\title{
Transcatheter MitraClip repair alters mitral annular geometry - device induced annular remodeling on three-dimensional echocardiography predicts therapeutic response
}

\author{
Jiwon Kim ${ }^{1 *+}$ (D), Maria Chiara Palumbo ${ }^{1,2+}$, Omar K. Khalique ${ }^{4}$, Lisa Q. Rong ${ }^{3}$, Razia Sultana ${ }^{1}$, Mukund Das ${ }^{1}$,
} Jennifer Jantz', Yasfumi Nagata ${ }^{5}$, Richard B. Devereux', Shing Chiu Wong', Geoffrey W. Bergman',

Robert A. Levine ${ }^{5}$, Mark B. Ratcliffe ${ }^{6,7}$ and Jonathan W. Weinsaft ${ }^{1}$

\begin{abstract}
Background: Echocardiography (echo) is widely used to guide therapeutic decision-making for patients being considered for MitraClip. Relative utility of two- (2D) and three-dimensional (3D) echo predictors of MitraClip response, and impact of MitraClip on mitral annular geometry, are uncertain.

Methods: The study population comprised patients with advanced (> moderate) MR undergoing MitraClip. Mitral annular geometry was quantified on pre-procedural 2D transthoracic echocardiography (TTE) and intra-procedural 3D transesophageal echocardiography (TEE); 3D TEE was used to measure MitraClip induced changes in annular geometry. Optimal MitraClip response was defined as $\leq$ mild MR on follow-up (mean $2.7 \pm 2.5$ months) post-procedure TTE.

Results: Eighty patients with advanced MR underwent MitraClip; 41\% had optimal response ( $\leq$ mild MR). Responders had smaller pre-procedural global left ventricular (LV) end-diastolic size and mitral annular diameter on 2D TTE (both $p \leq 0.01$ ), paralleling smaller annular area and circumference on 3D TEE (both $p=0.001$ ). Mitral annular size yielded good diagnostic performance for optimal MitraClip response (AUC 0.72, $p<0.01$ ). In multivariate analysis, sub-optimal MitraClip response was independently associated with larger pre-procedural mitral annular area on 3D TEE (OR 1.93 per $\mathrm{cm}^{2} / \mathrm{m}^{2}$ [Cl 1.19-3.13], $p=$ 0.007) and global LV end-diastolic volume on $2 \mathrm{D} T \mathrm{TTE}$ (OR 1.29 per $10 \mathrm{ml} / \mathrm{m}^{2}$ [Cl 1.02-1.63], $p=0.03$ ). Substitution of 2D TTE derived mitral annular diameter for 3D TEE data demonstrated a lesser association between pre-procedural annular size (OR 5.36 per $\mathrm{cm} / \mathrm{m}^{2}$ [Cl 0.95-30.19], $p=0.06$ ) and sub-optimal MitraClip response. Matched pre- and post-procedural TEE analyses demonstrated MitraClip to acutely decrease mitral annular area and circumference (all $p<0.001$ ) as well as mitral tenting height, area, and volume (all $p<0.05$ ): Magnitude of MitraClip induced reductions in mitral annular circumference on intra-procedural 3D TEE was greater among patients with, compared to those without, sub-optimal MitraClip response (>mild MR) on followup TTE $(p=0.017)$; greater magnitude of device-induced annular reduction remained associated with sub-optimal MitraClip response even when normalized for pre-procedure annular circumference $(p=0.028)$.

(Continued on next page)
\end{abstract}

\footnotetext{
* Correspondence: jik9027@med.cornell.edu

† Jiwon Kim and Maria Chiara Palumbo contributed equally to this work.

${ }^{1}$ Department of Medicine (Cardiology), Weill Cornell Medicine, 525 East 68th

Street, New York, NY 10021, USA

Full list of author information is available at the end of the article
}

(c) The Author(s). 2019 Open Access This article is distributed under the terms of the Creative Commons Attribution 4.0 International License (http://creativecommons.org/licenses/by/4.0/), which permits unrestricted use, distribution, and reproduction in any medium, provided you give appropriate credit to the original author(s) and the source, provide a link to the Creative Commons license, and indicate if changes were made. The Creative Commons Public Domain Dedication waiver (http://creativecommons.org/publicdomain/zero/1.0/) applies to the data made available in this article, unless otherwise stated. 
(Continued from previous page)

Conclusions: MitraClip alters mitral annular geometry as quantified by intra-procedural 3D TEE. Pre-procedural mitral annular dilation and magnitude of device-induced reduction in mitral annular size on 3D TEE are each associated with sub-optimal therapeutic response to MitraClip.

Keywords: MitraClip, 3D echocardiography, Mitral annulus

\section{Background}

Echocardiography (echo) is widely used to diagnose mitral regurgitation (MR) and assess its response to therapeutic interventions. MitraClip is the sole percutaneous device commercially approved in the United States to treat MR, and is increasingly utilized worldwide for this purpose - over 80, 000 patients have undergone MitraClip in the last decade [1]. Recurrent MR after MitraClip is a substantial problem. Studies have reported residual or recurrent ( $>$ mild) MR in over one third of patients undergoing MitraClip [2-5]. Even when implanted in controlled research settings, MitraClip response varies - as evidenced by two recent trials (MITRA$\mathrm{FR}, \mathrm{COAPT}$ ) that yielded conflicting results regarding impact of MitraClip on MR reduction and clinical outcomes [6, 7].

One reason for variable MR response to MitraClip stems from patient-specific differences in cardiac chamber remodeling. MitraClip is intended to reduce MR via focal leaflet coaptation. However, prior echo studies by our group and others have shown increased left ventricular (LV) size to augment risk for recurrent MR after MitraClip implantation [5], supporting the notion that remodeling indices beyond mitral valve anatomy impact therapeutic response. It is also possible that the device itself contributes to risk for MR recurrence. MitraClip induced leaflet tension may alter annular geometry - thus contributing to recurrent MR via distortion of valve coaptation and tethering of peri-annular myocardium. Consistent with this, computational modeling studies have shown MitraClip to augment leaflet stress adjacent to the device and also to affect broader aspects of the mitral apparatus - including decreased annular size and increased stretch (displacement) of periannular LV myocardium [8]. It remains unclear whether clinical application of MitraClip produces in vivo alterations in mitral annular geometry, and how such remodeling impacts patient outcomes.

This study tested impact of MitraClip on mitral annular remodeling. To do so, intra-procedural three-dimensional (3D) transesophageal echo (TEE) was used to quantify annular geometry prior to and immediately after MitraClip implantation. Transthoracic echo (TTE) was analyzed pre- and early (within 6 months) post-procedure to evaluate change in MR. Study goals were to test whether MitraClip acutely alters mitral annular geometry in a manner discernable via 3D TEE, and if magnitude of deviceinduced alterations in annular geometry stratifies MitraClip therapeutic response.

\section{Methods}

The study population comprised consecutive patients with advanced (>moderate) MR who underwent MitraClip at Weill Cornell Medicine (NY, NY) in whom intraprocedural TEE was available to evaluate annular geometry, and TTE was performed pre- and post- (1-6 months [target 6 months]) procedure to assess change in MR: No otherwise eligible patients were excluded based on procedural outcomes, imaging findings, or clinical indices.

Demographic data were collected in a standardized manner, including cardiac risk factors and medications. Procedural indices including number of MitraClip devices were also recorded. Ambulatory blood pressure, heart rate, and cardiac rhythm was measured at time of baseline and followup TTE. This analysis of pre-existing (retrospective) data for research purposes was approved by the Weill Cornell Institutional Review Board.

\section{Image acquisition}

To evaluate mitral annular and cardiac chamber geometry in relation to MitraClip response, data were derived from TEE and TTE, both of which were acquired via a standardized protocol:

- TEEs were acquired intra-procedure in a midesophageal view using Philips iE33 or EPIQ7 systems equipped with matrix array transducers. 3D images of the mitral apparatus including the annulus were optimized for coverage and gain using single beat acquisition (Zoom 3D); datasets were selected for analysis based on optimal discernment of the mitral annulus.

- TTEs were obtained using commercial equipment. Images were acquired in parasternal long, parasternal short, and apical 2-, 3-, and 4- chamber orientations. Color and pulsed wave Doppler was used to assess MR; continuous wave Doppler included assessment of tricuspid regurgitant velocity (to quantify pulmonary artery [PA] systolic pressure). 


\section{Image analysis}

\section{Three dimensional mitral annular geometry}

3D TEE analysis was performed using a semi-automated program (TomTEC 4D MV [Munich, Germany]) tailored for 3D mitral annular modeling. All mesurements were performed blinded to MitraClip response. A latesystolic frame was selected for annular tracking defined as the last systolic frame before mitral valve opening. Landmarks denoting anterior and posterior mitral annulus were identified on long orthogonal views followed by identification of the coaptation points of the aortic and mitral valves and apical posterior aspect of the aortic annulus. Semi-automated contours of the mitral annulus and mitral valve leaflets were then generated throughout the cardiac cycle. Contours were were manually edited via rotation around the mitral annulus to ensure accurate border segmentation. Calculated indices included valvular tenting area and height, mitral annular linear (antero-posterior, anterolateral-posteromedial) dimensions, as well as mitral annular area and circumference (see Fig. 1 for representative example).

\section{Chamber quantification}

LV chamber size, function, and mass were quantified on TTE based on linear dimensions in parasternal long axis orientation, concordant with established methods validated in prior research $[9,10]$. LV end-diastolic and end-systolic internal dimensions were measured at the level of mitral leaflet tips; mitral annular diameter was measured as the distance between annular insertion into the lateral LV wall and inferoseptum at LV end-diastole in apical 4-chamber orientation. LV mass was quantified using anteroseptal and posterior wall thickness, concordant with validated methods [11]. LV global longitudinal strain was calculated based on aggregate (2, 3, 4-chamber) long axis data in accordance with established methods previously applied by our group [12]. Left atrial (LA) area was quantified in apical 2- and 4-chamber orientation, for which results were used to quantify LA volume. LA global longitudinal and circumferential strain was quantified 4 chamber orientation; strain indices were derived using commercial software (TomTEC [Munich, Germany]), for which automated border detection was manually adjusted to ensure optimal tracking throughout the cardiac cycle.

\section{Mitral regurgitation}

MR severity was analyzed on TTE by dedicated ACC/AHA level III trained readers in a high-volume laboratory, for which expertise in MR quantification has been documented [13, 14]. MR was graded for study purposes using consensus guidelines based on an aggregate 4-point scale $(1=$ mild $-4=$ severe $)$ [15], for which primary components included vena contracta, regurgitant fraction, regurgitant volume, and effective regurgitant orifice area (EROA).

Optimal MitraClip response was defined as $\leq$ mild MR on follow-up TTE; a criterion concordant with prior surgical mitral repair literature [16], as well as MitraClip outcomes studies $[3,4]$.

\section{Statistical methods}

Comparisons between groups were made using Student's $\mathrm{t}$ test (expressed as mean \pm standard deviation) for continuous variables, and chi-square test for categorical variables. Bivariate correlation coefficients were used to evaluate associations between continuous variables. Univariable and multivariate modeling was performed via

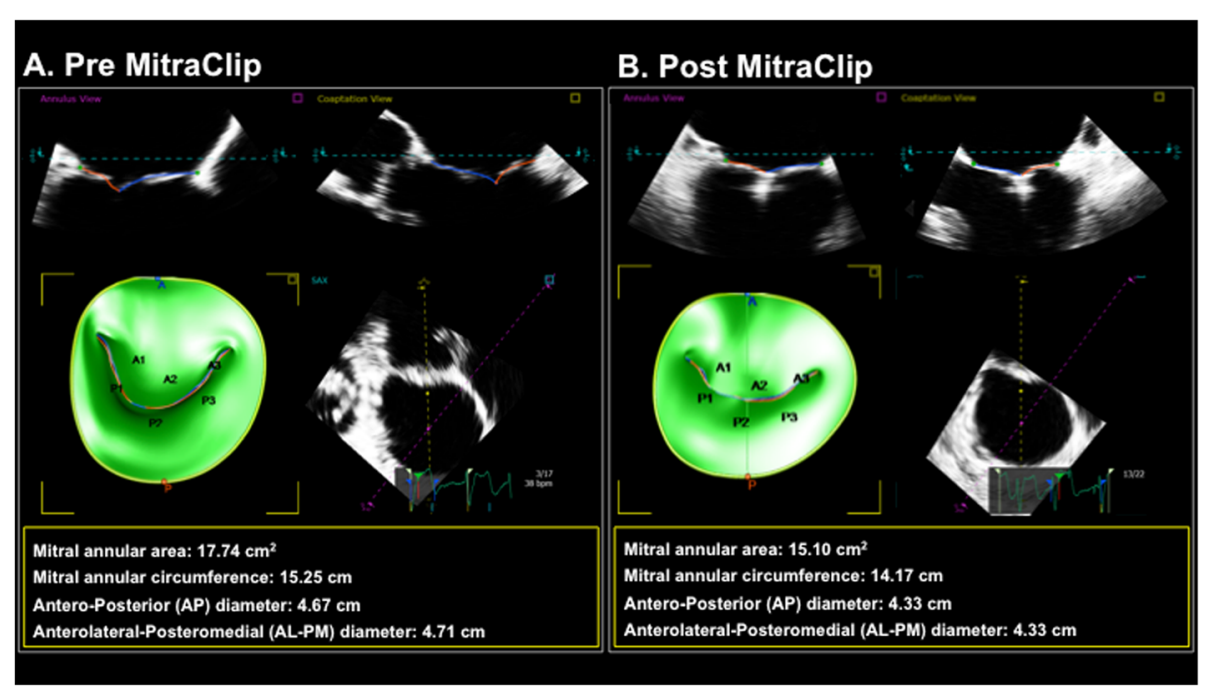

Fig. 1 MitraClip Induced Mitral Annular Remodeling on 3D Transesophageal Echo. Representative example of mitral apparatus remodeling parameters as quantified using intra-procedural 3D TEE. Note device-induced reductions in mitral annular circumference and area, paralleling post-procedure reductions in mitral annular linear indices 
binary logistic regression. Diagnostic utility of remodeling indices was evaluated in relation to MitraClip response using receiver operator characteristics curves, for which area under the curve (AUC) was used as an index of overall test performance. Statistical calculations were performed using SPSS 22.0 (SPSS Inc. [Chicago, IL]). Two-sided $p<0.05$ was considered indicative of statistical significance.

\section{Results}

\section{Population characteristics}

The study population comprised 80 patients with advanced (> moderate) MR who underwent MitraClip as well as pre- and post-procedure TTE to assess procedural durability, reflecting $78 \%$ of all patients who underwent this procedure at our site (Weill Cornell Medicine [NY, NY]) during the study interval (2013-19). MitraClip implantation employed the NTr device type in over three-fourths of cases (87\% NT/NTr; 13\% XTr). In nearly all cases (76/80), MR was deemed primarily degenerative; leading pathologies were prolapse, annular calcification, and valve thickening. However, concommitant conditions predisposing to mixed MR (with functional component) was common; $26 \%$ of patients had prior MI and $47 \%$ had systolic heart failure $(\mathrm{LVEF}<50 \%)$. All patients underwent
MitraClip without complications; a mean of $1.63 \pm 0.58$ devices were implanted per procedure (58\% had multiple devices implanted during the index intervention).

Follow-up TTE $(2.7 \pm 2.5$ months $)$ was performed to assess short term procedural response based on MR: Whereas nearly all (91\%) patients had some improvement in MR ( $\geq 1$ grade MR reduction), less than half (41\%) had optimal MitraClip response ( $\leq$ mild MR). Regarding thereapeutic response, follow-up data demonstrated MR trace/ absent in 10\% (33\% 1+ [mild] / 17\% 2+ [moderate]/ 30\% $3+$ [moderately-severe] / 10\% 4+ [severe]). Table 1 reports clinical characteristics over the overall study population, including comparisons between patients with and without optimal MitraClip response ( $\leq$ mild $[1+]$ MR). As shown, CAD and associated clinical risk factors for adverse LV chamber remodeling were highly common, but of similar prevalence between groups stratified based on MitraClip response (all $p=\mathrm{NS}$ ).

\section{MitraClip response in relation to chamber geometry}

Table 2 reports 2D TTE derived structural and functional parameters in relation to MitraClip response. Despite similar clinical profiles, results demonstrate that

Table 1 Clinical characteristics

\begin{tabular}{|c|c|c|c|c|}
\hline & Overall $(n=80)$ & $M C_{R S P}+{ }^{a}(n=33)$ & $M C_{\text {RSP }}-(n=47)$ & $p$ \\
\hline Age (year) & $79 \pm 10$ & $79 \pm 9$ & $80 \pm 10$ & 0.72 \\
\hline Male gender & $63 \%(50)$ & $64 \%(21)$ & $62 \%(29)$ & 0.86 \\
\hline Heart rate (bpm) & $70 \pm 12$ & $72 \pm 14$ & $70 \pm 11$ & 0.52 \\
\hline Systolic blood pressure $(\mathrm{mmHg})$ & $116 \pm 18$ & $119 \pm 18$ & $113 \pm 17$ & 0.17 \\
\hline Diastolic blood pressure (mmHg) & $65 \pm 12$ & $64 \pm 13$ & $66 \pm 10$ & 0.59 \\
\hline \multicolumn{5}{|l|}{ Atherosclerosis Risk Factors } \\
\hline Hypertension & $81 \%(65)$ & $88 \%(29)$ & $77 \%(36)$ & 0.20 \\
\hline Hypercholesterolemia & $66 \%(53)$ & $61 \%(20)$ & $70 \%(33)$ & 0.37 \\
\hline Diabetes mellitus & $29 \%(23)$ & $39 \%(13)$ & $21 \%(10)$ & 0.08 \\
\hline Tobacco use & $61 \%(49)$ & $55 \%(18)$ & $66 \%(31)$ & 0.30 \\
\hline Coronary Artery Disease & $54 \%(43)$ & $52 \%(17)$ & $55 \%(26)$ & 0.74 \\
\hline Prior Myocardial Infarction & $26 \%(21)$ & $21 \%(7)$ & $30 \%(14)$ & 0.39 \\
\hline Prior Revascularization & $41 \%(33)$ & $39 \%(13)$ & $43 \%(20)$ & 0.78 \\
\hline Atrial fibrillation/flutter & $39 \%(31)$ & $39 \%(13)$ & $38 \%(18)$ & 0.92 \\
\hline \multicolumn{5}{|l|}{ Cardiovascular Medications } \\
\hline Beta-blocker & $79 \%(63)$ & $82 \%(27)$ & $77 \%(36)$ & 0.57 \\
\hline ACE-Inhibitor/Angiotensin Receptor Blocker & $56 \%(45)$ & $52 \%(17)$ & $60 \%(28)$ & 0.47 \\
\hline Loop diuretic & $81 \%(65)$ & $85 \%(28)$ & $79 \%(37)$ & 0.49 \\
\hline HMG CoA-Reductase Inhibitor & $69 \%(55)$ & $70 \%(23)$ & $68 \%(32)$ & 0.88 \\
\hline Aspirin & $59 \%(47)$ & $52 \%(17)$ & $64 \%(30)$ & 0.27 \\
\hline \multicolumn{5}{|l|}{ Number of Clips Implanted } \\
\hline Mean & $1.63 \pm 0.58$ & $1.61 \pm 0.50$ & $1.64 \pm 0.64$ & 0.81 \\
\hline Multiple (> 1) & $46(57 \%)$ & $20(61 \%)$ & $26(55 \%)$ & 0.64 \\
\hline
\end{tabular}

${ }^{a}$ Optimal $M C_{R S P}$ defined as $\leq$ mild MR after MitraClip 
Table 2 Baseline imaging characteristics

\begin{tabular}{|c|c|c|c|c|}
\hline & Overall $(n=80)$ & $M C_{R S P}+{ }^{a}(n=33)$ & $M C_{\text {RSP }}-(n=47)$ & $\mathrm{p}$ \\
\hline \multicolumn{5}{|l|}{ Mitral Regurgitation } \\
\hline \multicolumn{5}{|l|}{ Regurgitant Severity } \\
\hline Regurgitant fraction (\%) & $56 \pm 16$ & $53 \pm 12$ & $58 \pm 19$ & 0.19 \\
\hline EROA $\left(\mathrm{cm}^{2}\right)$ & $0.6 \pm 0.3$ & $0.5 \pm 0.3$ & $0.6 \pm 0.3$ & 0.18 \\
\hline Regurgitant volume (ml) & $88 \pm 39$ & $80 \pm 35$ & $94 \pm 41$ & 0.13 \\
\hline \multicolumn{5}{|l|}{ Mitral Valve Morphology } \\
\hline Prolapse & $37 \%(30)$ & $39 \%(13)$ & $36 \%(17)$ & 0.77 \\
\hline Mitral annular calcification & $41 \%(33)$ & $45 \%(15)$ & $38 \%(18)$ & 0.52 \\
\hline Mitral valve thickening & $57 \%(46)$ & $55 \%(18)$ & $60 \%(28)$ & 0.65 \\
\hline Flail pathology & $14 \%(11)$ & $12 \%(4)$ & $15 \%(7)$ & 1.00 \\
\hline \multicolumn{5}{|l|}{ Left Ventricle } \\
\hline Ejection fraction (\%) & $49 \pm 15$ & $48 \pm 15$ & $49 \pm 15$ & 0.99 \\
\hline Global Longitudinal Strain (\%) & $15.8 \pm 5.3$ & $16.1 \pm 4.8$ & $15.3 \pm 5.7$ & 0.48 \\
\hline End-diastolic diameter (cm) & $6.0 \pm 0.8$ & $5.8 \pm 0.7$ & $6.2 \pm 0.8$ & 0.007 \\
\hline End-diastolic volume $\left(\mathrm{ml} / \mathrm{m}^{2}\right)$ & $104 \pm 30$ & $94 \pm 25$ & $111 \pm 31$ & 0.01 \\
\hline End-systolic diameter (cm) & $4.5 \pm 1.1$ & $4.3 \pm 1.0$ & $4.7 \pm 1.1$ & 0.10 \\
\hline End-systolic volume $\left(\mathrm{ml} / \mathrm{m}^{2}\right)$ & $56 \pm 32$ & $49 \pm 27$ & $61 \pm 34$ & 0.12 \\
\hline Mitral Annular Diameter & $3.36 \pm 0.49$ & $3.19 \pm 0.45$ & $3.48 \pm 0.49$ & 0.009 \\
\hline Myocardial mass $\left(\mathrm{g} / \mathrm{m}^{2}\right)$ & $112 \pm 31$ & $107 \pm 28$ & $116 \pm 33$ & 0.21 \\
\hline Relative wall thickness & $0.26 \pm 0.05$ & $0.28 \pm 0.05$ & $0.25 \pm 0.05$ & 0.02 \\
\hline \multicolumn{5}{|l|}{ Left Atrium } \\
\hline Diameter (cm) & $5.0 \pm 1.1$ & $4.8 \pm 1.0$ & $5.2 \pm 1.1$ & 0.10 \\
\hline 2-Chamber area $\left(\mathrm{cm}^{2}\right)$ & $32 \pm 12$ & $29.0 \pm 7.6$ & $34.5 \pm 13.3$ & 0.03 \\
\hline 4-Chamber area $\left(\mathrm{cm}^{2}\right)$ & $33 \pm 10$ & $28.6 \pm 7.4$ & $35.4 \pm 10.8$ & 0.003 \\
\hline Volume $\left(\mathrm{ml} / \mathrm{m}^{2}\right)$ & $73 \pm 37$ & $62 \pm 27$ & $82 \pm 41$ & 0.01 \\
\hline Global Circumferential Strain (\%) & $13.4 \pm 7.2$ & $15.5 \pm 7.6$ & $12.0 \pm 6.6$ & 0.03 \\
\hline Global Longitudinal Strain (\%) & $12.5 \pm 5.1$ & $14.1 \pm 5.7$ & $11.5 \pm 4.3$ & 0.02 \\
\hline Ejection Fraction (\%) & $35 \pm 15$ & $37 \pm 15$ & $33 \pm 16$ & 0.19 \\
\hline \multicolumn{5}{|l|}{ Right Ventricle } \\
\hline TAPSE (cm) & $1.8 \pm 0.6$ & $1.8 \pm 0.5$ & $1.8 \pm 0.6$ & 0.55 \\
\hline $\mathrm{S}^{\prime}(\mathrm{cm} / \mathrm{s})$ & $10.7 \pm 3.0$ & $10.3 \pm 2.7$ & $11.1 \pm 3.2$ & 0.23 \\
\hline Pulmonary artery systolic pressure $(\mathrm{mmHg})$ & $52 \pm 16$ & $55 \pm 18$ & $51 \pm 14$ & 0.21 \\
\hline
\end{tabular}

${ }^{a}$ Optimal $M C_{\text {RSP }}$ defined as $\leq$ mild MR after MitraClip

Data in bold are significant

MitraClip non-responders had more advanced chamber remodeling, as evidenced by increased mitral annular diameter and global LV size (both $p<0.01$ ). Regarding LA remodeling, results similarly demonstrated sub-optimal MitraClip to be associated with chamber dilation: LA area was, on average, more than 25\% larger among MitraClip non-responders - results were similar based on data acquired in 2- and 4-chamber orientation (both $p<0.05$ ), as well as calculated LA volume $(p=0.01)$. LA dilation was accompanied by impaired LA function; LA circumferential and longitundinal strain were both lower among MitraClip non-responders $(p<0.05)$ : circumferential $(r=$
$-0.47)$ and longitudinal strain $(r=-0.35$, both $p<0.001)$ were each negatively correlated with LA area.

Table 3 reports 3D mitral apparatus remodeling indices as quantified on TEE (available in $83 \%$ [ $n=$ 66]). As shown, 3D TEE results paralleled those of 2D TTE with respect to associations between adverse remodeling and MitraClip response: Mitral annular area, circumference, and diameter were all equivalently larger among patients with suboptimal MitraClip response $(p<0.001)$, paralleling a trend towards increased tenting height $(p=0.08)$ in suboptimal responders. 
Table 3 Pre-procedural transesophageal echo mitral annular indices relation to MitraClip response

\begin{tabular}{|c|c|c|c|}
\hline & $M C_{R S P}+{ }^{a}(n=26)$ & $\mathrm{MC}_{\mathrm{RSP}}-(n=40)$ & $\mathrm{p}$ \\
\hline Mitral Annular Area $\left(\mathrm{cm}^{2}\right)$ & $12.85 \pm 1.90$ & $15.28 \pm 3.48$ & 0.001 \\
\hline$\left(\mathrm{cm}^{2} / \mathrm{m}^{2}\right)$ & $7.07 \pm 1.02$ & $8.49 \pm 2.06$ & $<0.001$ \\
\hline Annular Circumference $(\mathrm{cm})$ & $13.10 \pm 0.97$ & $14.27 \pm 1.54$ & 0.001 \\
\hline$\left(\mathrm{cm} / \mathrm{m}^{2}\right)$ & $7.23 \pm 0.83$ & $7.95 \pm 1.25$ & 0.01 \\
\hline Antero-Posterior Diameter $(\mathrm{cm})$ & $3.76 \pm 0.36$ & $4.12 \pm 0.53$ & 0.003 \\
\hline Anterolateral-Posteromedial Diameter $(\mathrm{cm})$ & $4.20 \pm 0.38$ & $4.54 \pm 0.51$ & 0.005 \\
\hline Annular Height (cm) & $0.72 \pm 0.17$ & $0.82 \pm 0.24$ & 0.06 \\
\hline Nonplanar Angle $\left(^{\circ}\right)$ & $154.05 \pm 9.98$ & $150.06 \pm 12.40$ & 0.17 \\
\hline Tenting Volume $\left(\mathrm{cm}^{3}\right)$ & $3.54 \pm 1.86$ & $4.22 \pm 1.70$ & 0.14 \\
\hline Tenting Area $\left(\mathrm{cm}^{2}\right)$ & $1.80 \pm 0.78$ & $2.09 \pm 0.75$ & 0.13 \\
\hline Tenting Height (mm) & $8.63 \pm 3.06$ & $9.92 \pm 2.79$ & 0.08 \\
\hline Anterior Leaflet Angle $\left(^{\circ}\right)$ & $36.53 \pm 14.86$ & $35.05 \pm 13.49$ & 0.68 \\
\hline Posterior Leaflet Angle $\left(^{\circ}\right)$ & $18.97 \pm 6.45$ & $21.90 \pm 7.39$ & 0.10 \\
\hline
\end{tabular}

${ }^{\mathrm{a}}$ Optimal $\mathrm{MC}_{\mathrm{RSP}}$ defined as $\leq$ mild MR after MitraClip

Data in bold are significant

\section{Remodeling based predictors of MitraClip response}

Regression analyses were used to test relative utility of remodeling indices for prediction of MitraClip response. As shown in Table 4, univariate analyses demonstrated likelihood of optimal response $(\leq$ mild $M R)$ at follow-up to decrease in relation to magnitude of increased mitral annular size on pre-procedural TEE, irrespective of whether quantified based on annular area (OR 1.93 per $\mathrm{cm}^{2} / \mathrm{m}^{2}$ [CI 1.22-3.06]), circumference (OR 1.96 per $\mathrm{cm} / \mathrm{m}^{2}$ [CI 1.12-3.41]), or corresponding $2 \mathrm{D}$ area and linear based indices (all $p<0.05$ ): Predictive utility of TEE indices of mitral annular size were similar, as evidenced by similar overall diagnostic performance (AUC 0.65-0.72). Table 4 also demonstrates TTE derived

Table 4 Structural predictors of sub-optimal MitraClip response

\begin{tabular}{|c|c|c|c|c|c|c|}
\hline & Odds Ratio (95\% Confidence Interval) & $\mathrm{p}$ & AUC & Cutoff & Sensitivity & Specificity \\
\hline \multicolumn{7}{|l|}{ 3D Transesophogeal Echo } \\
\hline Mitral Annular Area $\left(\mathrm{cm}^{2} / \mathrm{m}^{2}\right)$ & $1.93(1.22-3.06)$ & 0.005 & $0.72(0.59-0.84)$ & 6.72 & $82 \%$ & $46 \%$ \\
\hline Annular Circumference $\left(\mathrm{cm} / \mathrm{m}^{2}\right)$ & $1.96(1.12-3.41)$ & 0.02 & $0.65(0.52-0.79)$ & 6.83 & $82 \%$ & $42 \%$ \\
\hline Antero-Posterior Diameter (cm) & $7.16(1.72-29.85)$ & 0.007 & $0.72(0.60-0.85)$ & 3.77 & $80 \%$ & $54 \%$ \\
\hline Anterolateral-Posteromedial Diameter (cm) & $6.35(1.58-25.44)$ & 0.009 & $0.70(0.57-0.82)$ & 4.03 & $87 \%$ & $38 \%$ \\
\hline Annular Height (cm) & $8.27(0.73-93.28)$ & 0.09 & $0.63(0.50-0.77)$ & 0.62 & $82 \%$ & $35 \%$ \\
\hline Tenting Volume $\left(\mathrm{cm}^{3}\right)$ & $1.26(0.93-1.70)$ & 0.14 & $0.64(0.50-0.78)$ & 2.69 & $82 \%$ & $38 \%$ \\
\hline Tenting Area $\left(\mathrm{cm}^{2}\right)$ & $1.70(0.85-3.38)$ & 0.13 & $0.63(0.49-0.77)$ & 1.51 & $80 \%$ & $38 \%$ \\
\hline Tenting Height (mm) & $1.17(0.98-1.40)$ & 0.09 & $0.64(0.50-0.79)$ & 7.80 & $87 \%$ & $50 \%$ \\
\hline Anterior Leaflet Angle $\left(^{\circ}\right)$ & $0.99(0.96-1.03)$ & 0.67 & $0.50(0.35-0.64)$ & 24.55 & $82 \%$ & $27 \%$ \\
\hline Posterior Leaflet Angle $\left(^{\circ}\right)$ & $1.06(0.99-1.15)$ & 0.11 & $0.64(0.50-0.79)$ & 16.05 & $85 \%$ & $46 \%$ \\
\hline Nonplanar Angle $\left(^{\circ}\right)$ & $0.97(0.93-1.01)$ & 0.17 & $0.40(0.26-0.54)$ & 140.40 & $82 \%$ & $11 \%$ \\
\hline \multicolumn{7}{|l|}{ 2D Transthoracic Echo } \\
\hline Mitral Annular Diameter $\left(\mathrm{cm} / \mathrm{m}^{2}\right)$ & $6.50(1.27-33.20)$ & 0.02 & $0.64(0.51-0.76)$ & 1.67 & $81 \%$ & $46 \%$ \\
\hline LV End-diastolic volume $\left(\mathrm{ml} / \mathrm{m}^{2}\right)^{a}$ & $1.25(1.04-1.50)$ & 0.01 & $0.67(0.55-0.79)$ & 8.46 & $81 \%$ & $39 \%$ \\
\hline LA Volume $\left(\mathrm{ml} / \mathrm{m}^{2}\right)^{\mathrm{a}}$ & $1.22(1.03-1.44)$ & 0.02 & $0.68(0.56-0.80)$ & 5.41 & $83 \%$ & $54 \%$ \\
\hline LA 2-Chamber area $\left(\mathrm{cm}^{2}\right)$ & $1.06(1.00-1.11)$ & 0.04 & $0.64(0.52-0.77)$ & 24.65 & $87 \%$ & $33 \%$ \\
\hline LA 4-Chamber area $\left(\mathrm{cm}^{2}\right)$ & $1.09(1.02-1.16)$ & 0.006 & $0.68(0.56-0.80)$ & 27.95 & $81 \%$ & $54 \%$ \\
\hline
\end{tabular}


cardiac chamber remodeling indices to be associated with MitraClip response: LV (OR 1.25 per $10 \mathrm{ml} / \mathrm{m}^{2}$ [CI 1.04-1.50]) and LA (OR 1.22 per $10 \mathrm{ml} / \mathrm{m}^{2}$ [CI 1.031.44]) volume were each associated $(p<0.05)$ with greater residual MR (>mild) after MitraClip implantation. Similarly, increased mitral annular diameter as quantified on TTE was associated with MitraClip response $(p=0.02)$, as was the case for annular size on intra-oprocedural TEE imaging $(p<0.01)$.

Multivariate analysis was used to further test the association of pre-procedural remodeling indices with MitraClip response. As shown in Table 5A and B, sub-optimal MitraClip response was independently associated with increased mitral annular area on 3D TEE (OR 1.93 per $\mathrm{cm}^{2} / \mathrm{m}^{2}$ [CI 1.19-3.13], $p=0.007$ ) as well as global LV volume as quantified by 2D TTE (OR 1.29 per $10 \mathrm{ml} / \mathrm{m}^{2}$ [CI 1.02-1.63], $p=0.03$ ). Substitution of 2D TTE derived mitral annular diameter (in place of corresponding 3D TEE area) weakened the predictive model $\left(x^{2} 17.22 \rightarrow\right.$ 10.87), and demonstrated a lesser association between pre-procedural annular diameter $(p=0.06)$ and greater residual MR (>mild) after MitraClip implantation.

\section{MitraClip induced annular remodeling}

Given our observed association between pre-procedural mitral annular area and therapeutic response, 3D TEE data were further analyzed to test whether MitraClip acutely altered annular geometry, and whether such alterations predicted procedural success. Table 6 reports preand post-procedural mitral annular geometry among the overall cohort, demonstrating device-induced reductions in mitral annular area and circumference, as well as valvular tenting area and volume (all $p<0.001$ ): Fig. 1 provides a representative example of MitraClip induced alterations in annular geometry. Of note, Table 6 also demonstrates that MitraClip induced changes in annular geometry (area, circumferential, and linear indices) generally were similar among patient subgroups with mitral prolapse $(n=19)$ and prominent annular calcification $(n=25)$ - although device-induced reductions in tenting indices were nonsignificant in patients with prolapse.

As illustrated in Fig. 2, magnitude of MitraClip induced reductions in annular circumference on intra-procedural TEE was nearly 2 -fold greater among patients with, compared to those without, sub-optimal MitraClip response ( $>$ mild MR) on followup TTE $(0.73 \pm 0.58$ vs. $0.34 \pm 0.50 \mathrm{~cm}, p=0.017)$; greater magnitude of MitraClip induced annular reduction remained significantly associated with MitraClip response even when normalized for pre-procedural circumference $(p=0.028)$. Similarly, 3D TEE analysis demonstrated intraprocedural device-induced reductions in mitral annular area to be greater among patients with sub-optimal MitraClip response $\left(1.59 \pm 1.26\right.$ vs. $\left.0.89 \pm 0.98 \mathrm{~cm}^{2}, p=0.038\right)$ on follow-up TTE.

\section{Discussion}

This is the first study to examine the impact of MitraClip on mitral annular remodeling - findings provide new insights regarding mechanistic determinants of therapeutic response, as well as novel echo-based parameters to guide procedural decision-making. Results demonstrate that MitraClip alters annular geometry in regions beyond the device itself, as evidenced by acute device-induced reductions in annular circumference and area $(p<0.001)$ as quantified on intra-procedural 3D TEE. Consistent with the notion that remodeling factors beyond the device impact device efficacy, pre-procedural mitral annular and LV chamber size each independently predicted sub-optimal MitraClip response; substitution of 3D TEE derived mitral annular area for annular diameter on 2D TTE strengthened the predictive model. Magnitude of intra-procedural device-induced reduction in mitral annular size was greater among patients with sub-optimal MitraClip response (>mild MR) on followup, as evidenced by greater absolute and relative reductions in mitral annular circumference (both $p<0.05$ ).

Regarding mechanism, it should be noted that MitraClip is intended to reduce MR via device-induced leaflet coaptation

Table 5 Multivariate models for prediction of sub-optimal MitraClip response

\begin{tabular}{|c|c|c|}
\hline $5 \mathrm{~A}$. & $\begin{array}{l}\text { Multivariate Regression } \\
\text { Model chi square }=17.22, p<0.001\end{array}$ & \\
\hline Variable & Odds Ratio (95\% Confidence Interval) & $\mathrm{p}$ \\
\hline Mitral Annular Area $\left(\mathrm{cm}^{2} / \mathrm{m}^{2}\right)$ & $1.93(1.19-3.13)$ & 0.007 \\
\hline LV End Diastolic Volume $\left(\mathrm{ml} / \mathrm{m}^{2}\right)^{\mathrm{a}}$ & $1.29(1.02-1.63)$ & 0.03 \\
\hline 5B. & $\begin{array}{l}\text { Multivariate Regression } \\
\text { Model chi square }=10.87, p=0.004\end{array}$ & \\
\hline Variable & Odds Ratio (95\% Confidence Interval) & $p$ \\
\hline Mitral Annular Diameter $\left(\mathrm{cm} / \mathrm{m}^{2}\right)$ & $5.36(0.95-30.19)$ & 0.06 \\
\hline LV End Diastolic Volume $\left(\mathrm{ml} / \mathrm{m}^{2}\right)^{\mathrm{a}}$ & $1.23(1.02-1.49)$ & 0.03 \\
\hline
\end{tabular}


Table 6 MitraClip induced annular remodeling

\begin{tabular}{|c|c|c|c|c|}
\hline & Pre & Post & $\Delta$ & $p$ \\
\hline \multicolumn{5}{|l|}{$\overline{\text { Overall }^{\circledR}}$} \\
\hline Mitral Annular Area $\left(\mathrm{cm}^{2}\right)$ & $14.20 \pm 2.97$ & $12.88 \pm 2.80$ & $-1.31 \pm 1.20$ & $<0.001$ \\
\hline$\left(\mathrm{cm} / \mathrm{m}^{2}\right)$ & $7.75 \pm 1.60$ & $7.02 \pm 1.42$ & $-0.73 \pm 0.65$ & $<0.001$ \\
\hline Annular Circumference (cm) & $13.73 \pm 1.36$ & $13.15 \pm 1.35$ & $-0.57 \pm 0.58$ & $<0.001$ \\
\hline$\left(\mathrm{cm} / \mathrm{m}^{2}\right)$ & $7.53 \pm 1.07$ & $7.21 \pm 0.98$ & $-0.32 \pm 0.32$ & $<0.001$ \\
\hline Antero-Posterior Diameter (cm) & $3.96 \pm 0.44$ & $3.61 \pm 0.42$ & $-0.35 \pm 0.25$ & $<0.001$ \\
\hline Anterolateral-Posteromedial Diameter (cm) & $4.37 \pm 0.48$ & $4.32 \pm 0.48$ & $-0.05 \pm 0.29$ & 0.20 \\
\hline Annular Height (cm) & $0.77 \pm 0.21$ & $0.71 \pm 0.20$ & $-0.06 \pm 0.21$ & 0.06 \\
\hline Tenting Volume $\left(\mathrm{cm}^{3}\right)$ & $4.07 \pm 1.90$ & $3.35 \pm 1.58$ & $-0.72 \pm 0.95$ & $<0.001$ \\
\hline Tenting Area $\left(\mathrm{cm}^{2}\right)$ & $2.01 \pm 0.84$ & $1.71 \pm 0.65$ & $-0.29 \pm 0.57$ & 0.001 \\
\hline Tenting Height (mm) & $9.44 \pm 3.17$ & $8.58 \pm 2.51$ & $-0.86 \pm 2.45$ & 0.015 \\
\hline Anterior Leaflet Angle $\left(^{\circ}\right)$ & $36.12 \pm 13.88$ & $34.32 \pm 13.86$ & $-1.80 \pm 9.96$ & 0.20 \\
\hline Posterior Leaflet Angle $\left(^{\circ}\right)$ & $21.05 \pm 7.79$ & $22.06 \pm 6.61$ & $1.02 \pm 6.59$ & 0.27 \\
\hline Nonplanar Angle $\left(^{\circ}\right)$ & $151.60 \pm 11.99$ & $148.03 \pm 15.46$ & $-3.57 \pm 14.67$ & 0.09 \\
\hline \multicolumn{5}{|l|}{ Mitral Prolapse } \\
\hline Mitral Annular Area $\left(\mathrm{cm}^{2}\right)$ & $14.37 \pm 3.26$ & $13.06 \pm 3.40$ & $-1.31 \pm 0.96$ & $<0.001$ \\
\hline$\left(\mathrm{cm} / \mathrm{m}^{2}\right)$ & $7.77 \pm 1.72$ & $7.04 \pm 1.66$ & $-0.73 \pm 0.58$ & $<0.001$ \\
\hline Annular Circumference (cm) & $13.84 \pm 1.45$ & $13.29 \pm 1.54$ & $-0.55 \pm 0.48$ & $<0.001$ \\
\hline$\left(\mathrm{cm} / \mathrm{m}^{2}\right)$ & $7.52 \pm 1.12$ & $7.21 \pm 1.00$ & $-0.31 \pm 0.29$ & $<0.001$ \\
\hline Antero-Posterior Diameter (cm) & $3.94 \pm 0.46$ & $3.57 \pm 0.49$ & $-0.37 \pm 0.27$ & $<0.001$ \\
\hline Anterolateral-Posteromedial Diameter (cm) & $4.40 \pm 0.55$ & $4.38 \pm 0.57$ & $-0.02 \pm 0.28$ & 0.77 \\
\hline Annular Height (cm) & $0.79 \pm 0.22$ & $0.72 \pm 0.19$ & $-0.07 \pm 0.18$ & 0.13 \\
\hline Tenting Volume $\left(\mathrm{cm}^{3}\right)$ & $2.59 \pm 1.02$ & $2.32 \pm 1.27$ & $-0.27 \pm 0.86$ & 0.22 \\
\hline Tenting Area $\left(\mathrm{cm}^{2}\right)$ & $1.38 \pm 0.60$ & $1.32 \pm 0.57$ & $-0.06 \pm 0.62$ & 0.69 \\
\hline Tenting Height (mm) & $7.53 \pm 3.19$ & $7.48 \pm 2.55$ & $-0.05 \pm 2.82$ & 0.94 \\
\hline Anterior Leaflet Angle $\left(^{\circ}\right)$ & $25.66 \pm 10.16$ & $27.19 \pm 11.58$ & $1.54 \pm 10.47$ & 0.53 \\
\hline Posterior Leaflet Angle $\left(^{\circ}\right)$ & $20.24 \pm 10.11$ & $21.85 \pm 8.65$ & $1.61 \pm 8.31$ & 0.41 \\
\hline Nonplanar Angle $\left({ }^{\circ}\right)$ & $149.44 \pm 13.64$ & $144.52 \pm 17.69$ & $-4.92 \pm 16.00$ & 0.20 \\
\hline \multicolumn{5}{|l|}{ Mitral Annular Calcification } \\
\hline Mitral Annular Area $\left(\mathrm{cm}^{2}\right)$ & $15.05 \pm 3.57$ & $13.58 \pm 3.29$ & $-1.47 \pm 1.33$ & $<0.001$ \\
\hline$\left(\mathrm{cm} / \mathrm{m}^{2}\right)$ & $8.28 \pm 1.82$ & $7.45 \pm 1.61$ & $-0.82 \pm 0.74$ & $<0.001$ \\
\hline Annular Circumference (cm) & $14.11 \pm 1.58$ & $13.48 \pm 1.49$ & $-0.63 \pm 0.58$ & $<0.001$ \\
\hline$\left(\mathrm{cm} / \mathrm{m}^{2}\right)$ & $7.81 \pm 1.18$ & $7.46 \pm 1.06$ & $-0.35 \pm 0.33$ & $<0.001$ \\
\hline Antero-Posterior Diameter (cm) & $4.08 \pm 0.50$ & $3.75 \pm 0.46$ & $-0.33 \pm 0.26$ & $<0.001$ \\
\hline Anterolateral-Posteromedial Diameter $(\mathrm{cm})$ & $4.49 \pm 0.59$ & $4.36 \pm 0.56$ & $-0.12 \pm 0.27$ & 0.03 \\
\hline Annular Height (cm) & $0.79 \pm 0.20$ & $0.73 \pm 0.22$ & $-0.07 \pm 0.22$ & 0.12 \\
\hline Tenting Volume $\left(\mathrm{cm}^{3}\right)$ & $4.23 \pm 1.99$ & $3.46 \pm 1.61$ & $-0.77 \pm 0.93$ & 0.001 \\
\hline Tenting Area $\left(\mathrm{cm}^{2}\right)$ & $1.97 \pm 0.91$ & $1.72 \pm 0.65$ & $-0.25 \pm 0.62$ & 0.055 \\
\hline Tenting Height (mm) & $8.86 \pm 3.42$ & $8.38 \pm 2.69$ & $-0.48 \pm 2.67$ & 0.38 \\
\hline Anterior Leaflet Angle $\left(^{\circ}\right)$ & $31.70 \pm 12.63$ & $31.41 \pm 12.22$ & $-0.30 \pm 9.88$ & 0.88 \\
\hline Posterior Leaflet Angle $\left(^{\circ}\right)$ & $20.08 \pm 8.88$ & $21.62 \pm 7.63$ & $1.54 \pm 7.62$ & 0.32 \\
\hline Nonplanar Angle $\left(^{\circ}\right)$ & $150.67 \pm 11.12$ & $146.64 \pm 16.80$ & $-4.03 \pm 14.20$ & 0.17 \\
\hline
\end{tabular}

apre and post-procedural 3D TEE data available in 51 patients (77\% of patients with TEE) Data in bold are significant 


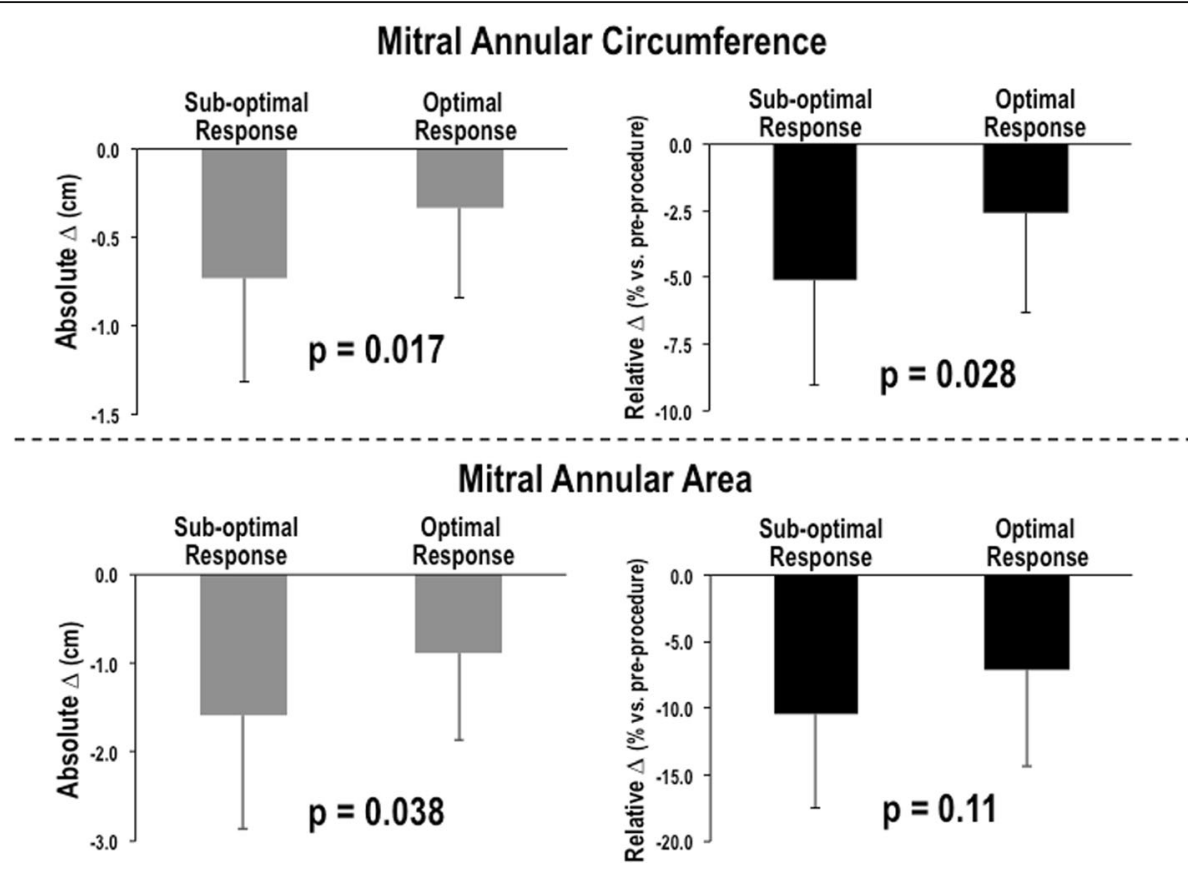

Fig. 2 MitraClip Induced Annular Remodeling in Relation to MR Response. Magnitude of acute MitraClip induced reduction in mitral annular circumference (top) and area (bottom), as quantified using intra-procedural TEE, among patients stratified based on optimal MitraClip response ( $\leq$ mild MR) as assessed on followup ambulatory TTE. Left: Greater magnitude of device-induced annular remodeling (reduced size) was observed among patients with sub-optimal MitraClip response, irrespective of whether quantified based on absolute change (pre - post) in 3D annular circumference or area (both $p<0.05$ ). Right: Similar results were obtained when examining relative change ([pre - post] / pre) in annular remodeling indices. Data shown as mean \pm standard deviation

- providing a nidus for increased leaflet stress and tension on peri-annular myocardium. In this context, our finding that MitraClip acutely reduced annular size supports the notion that tensile forces exerted by the device are sufficient to alter mitral apparatus geometry in regions beyond the clipped valve leaflets themselves, and that 3D TEE is sufficient to discern the remodeling effects of such tensile forces. Our finding of an association between pre-procedural dilation and MR recurrence also suggests that MitraClip-induced tensile forces may ultimately be insufficient in the context of marked annular or LV remodeling - a setting in which intrinsic resistance forces may be augmented, resulting in suboptimal MitraClip response. While exact causality for MR recurrence cannot be discerned from our current results, it is also possible that MitraClip induced annular remodeling alters valve coaptation geometry, augments angular displacement between the mitral valves and sub-valvular apparatus, and/or induces myocardial tethering - each of which can contribute to MR. Consistent with the latter, recent computational modeling data from our group demonstrated that MitraClip augmented leaflet stress immediately adjacent to the device as well as LV myocardial stretch adjacent to the mitral annulus: [8] Our current results provide proof of concept that device-induced annular remodeling occurs in vivo, severity of which can be discerned on 3D TEE as a means of predicting therapeutic response to MitraClip. Whereas a large majority (70/80) of our study population underwent repair using the MitraClip NT/NTr device, our finding of an impact of MitraClip on annular remodeling is of relevance in context of new modifications of the device (XTr) that grasp a wider amount of leaflet tissue, and would thus be expected to apply a greater magnitude of force on the mitral annulus. It is also possible that greater magnitude of leaflet grasp would better resist tethering forces exerted by sub-valvular myocardium, and thus provide a more durable reduction of MR. In this context, future studies are warrented to test whether pattern and magnitude of induced annular remodeling are similar irrespective of MitraClip device type, and whether specific types of MitraClip mitigate or augment the impact of device-induced annular remodeling on postprocedure MR.

Our current results build upon a growing body of literature which have demonstrated chamber dilation to predict prognosis and therapeutic success among patients undergoing MitraClip. Large scale MitraClip registry data comprised primarily (91\%) of patients with degenerative MR have also shown increased LV enddiastolic diameter to predict decreased likelihood of procedural success as assessed at time of hospital discharge [17]. Among patients with functional MR, differences in cardiac chamber remodeling may explain conflicting results of two recent MitraClip clinical trials (MITRA-FR, 
COAPT) $[6,7] . \mathrm{LV}$ chamber size was over $25 \%$ larger in MITRA-FR vs. COAPT patients, paralleling a $>3$-fold increase in recurrent severe MR: MITRA-FR reported MitraClip to have no impact on outcomes, whereas MR reduction in COAPT was accompanied by heart failure and mortality benefit. As further evidence of the importance of LV remodeling as a determinant of MitraClip response, prior research from our group (encompassing 67 patients included in the current cohort) showed increased LV size to be associated with risk for suboptimal MitraClip response (>mild MR): [5] However, the relative utility of $2 \mathrm{D}$ and $3 \mathrm{D}$ derived mitral annular size independent of LV chamber volume, impact of the device on annular remodeling, as well as intraprocedural annular remodeling as a determinant of therapeutic response were not tested - providing key rationales for the current study.

Regarding imaging, our data highlight the utility of 3D echo as a pre-procedural planning tool to guide patient selection and predict therapeutic therapeutic response to MitraClip. Our results extend upon recent data showing mitral annular geometry on 3D TEE to stratify likelihood of MR reduction following MitraClip implantation. Among a cohort of 31 patients with degenerative MR, Oguz et al. reported that increased pre-procedural tenting height and volume on 3D TEE were each associated with risk for sub-optimal MR reduction [18]. Whereas our results found increased tenting height to be generally associated with suboptimal response, predictive value of this parameter was less (OR 1.17 per mm [CI 0.98-1.40], $p=0.09$ ) than that of annular circumference or area (both $p<0.01$ ). While reasons for this difference are uncertain, we speculate that it may stem from more advanced LV remodeling in our study population as evidenced by increased LV chamber size in our population ( $\mathrm{LV}$ end diastolic diameter $6.0 \pm 0.8 \mathrm{~cm}$ ) compared to that studied by Oguz et al. $(5.8 \pm 0.7 \mathrm{~cm})$ : [18] Increased LV chamber size could potentially alter loading forces on the mitral valve, resulting in greater importance of pre-procedural mitral annular geometry as a determinant of MitraClip response. It is also possible that differences in valve pathology and/or myocardial substrate (i.e. fibrosis) could modify predictive utility of different mitral apparatus parameters. Given our sample size $(n=$ 80) and the fact that device-induced annular remodeling is intrinsically related to pre-procedural chamber geometry, our analyses were insufficiently powered to test whether baseline LV chamber size and device-induced remodeling were independent predictors of MitraClip response, as well as whether pre-procedural LV geometry, myocardial substrate (fibrosis), and device-induced annular remodeling synergistically impacted MR recurrence after MitraClip. Further, larger scale, research is warranted to test the relative prognostic utility of valvular and annular remodeling indices - inclusive of device-induced changes in annular geometry - as predictors of MitraClip response.

Several limitations should be noted. First, our study defined optimal MitraClip response using a binary partition for MR $(\leq$ mild $[1+])$, rather than a single quantitative measure. This approach is consistent with that employed in several prior studies in which greater severity of MR after MitraClip was shown to confer adverse prognosis $[3,4]$. Despite this, a variety of cutoffs for adequate MR reduction have been used in prior MitraClip studies, which may explain variable rates of recurrent MR. [2-4, 19-22] Of note, procedural success among our cohort $(41 \%)$ was near equivalent to that among degenerative MR patients undergoing MitraClip in the the EVEREST II trial, in which $43 \%$ of patients had $\leq$ mild MR at 1 year followup [2]. It is also important to recognize that follow-up was performed at a single time point after MitraClip, and that TTE was not performed immediately after device implantation. Accordingly, our analyses were unable to test relative contributions of residual MR and device-induced annular remodeling as determinants of MitraClip response - further studies employing serial imaging and more precise metrics of annular/myocardial stretch are necessary to expand on results of the current study. Finally, it should be noted that TEE was aquired using 3D technology, whereas TTE was limited to 2D. In this context, it is uncertain whether limits of TTE (i.e. lesser predictive utility of TTE quantified mitral annular size) stem from suboptimal mitral annular visualization by TTE, or the $2 \mathrm{D}$ approach used for data aquistion. Given that TEE is invasive, further research is warranted to test whether $3 \mathrm{D}$ TTE provides equivalent utility for predicting MitraClip therapeutic response.

\section{Conclusions}

This study demonstrates that MitraClip alters mitral annular geometry, and that magnitude of intra-procedural reduction in annular size is associated with sub-optimal device response (> mild MR). Future larger scale studies are warranted to further discern mechanism by which device-induced annular remodeling drives recurrent MR after MitraClip, and test clinical utility of 3D echo guided MR therapeutic strategies paired to pattern and magnitude of mitral apparatus remodeling.

\footnotetext{
Abbreviations

2D: Two-dimensional; 3D: Three-dimensional; CAD: Coronary artery disease; EROA: Effective regurgitant orifice area; LA: Left atrial; LV: Left ventricle; left ventricular; LVEF: Left ventricular ejection fraction; MI: Myocardial infarction; MR: Mitral regurgitation; PA: Pulmonary artery; TEE: Transesophageal echocardiography; TTE: Transthoracic echocardiography
}

Acknowledgements None 


\section{Authors' contributions}

JWW, JK, MCP, and RBD were involved in the design of the study and collection, analysis, and interpretation of data and were major contributors in writing the manuscript. JJ, OKK, RAL, MBR, RS, MD were involved in the analysis and interpretation of data and in writing the manuscript. SCW, GWB, $L Q R$, and $Y N$ were involved interpretation of data and in writing the manuscript. All authors read and approved the final manuscript.

\section{Funding}

National Institutes of Health 1 K23 HL140092 (JK), 1R01HL128278 (JWW, RAL, MR), 1R01HL141917 (RAL).

\section{Availability of data and materials}

The data, analytic methods, and study materials will be made available to other researchers for purposes of reproducing the results or replicating the procedure, upon request (contingent on approval of the Weill Cornell Institutional Review Board and assurance of data de-identification).

\section{Ethics approval and consent to participate}

This protocol was approved by the Weill Cornell Institutional Review Board, which approved analysis of pre-existing data for research purposes. Informed consent for use of research data was waived, as this was a retrospective study.

\section{Consent for publication}

Not applicable (patient identifiers not included in manuscript).

\section{Competing interests}

Dr. Ratcliffe previously performed a computational modeling study of the Ventouch device (for ventricular reshaping in functional mitral regurgitation) under contract to Mardil Medical Inc.

\section{Author details}

'Department of Medicine (Cardiology), Weill Cornell Medicine, 525 East 68th Street, New York, NY 10021, USA. ${ }^{2}$ Department of Cardiothoracic Surgery, Weill Cornell Medicine, New York, NY, USA. ${ }^{3}$ Department of Anesthesiology, Weill Cornell Medicine, New York, NY, USA. ${ }^{4}$ Division of Cardiology, Columbia University Medical Center, New York, NY, USA. ${ }^{5}$ Division of Cardiology -Massachusetts General Hospital, Harvard Medical School, Boston, MA, USA. ${ }^{6}$ Department of Bioengineering, University of California, San Francisco, USA. 7 Veterans Affairs Medical Center, San Francisco, CA, USA.

Received: 7 October 2019 Accepted: 11 December 2019

Published online: 26 December 2019

\section{References}

1. Abbott receives FDA approval for expanded indication for MitraClip ${ }^{T M}$ device. 2019. https://abbott.mediaroom.com/2019-03-14-Abbott-ReceivesFDA-Approval-for-Expanded-Indication-for-MitraClip-TM-Device.

2. Mauri L, Foster E, Glower DD, Apruzzese P, Massaro JM, Herrmann HC, Hermiller J, Gray W, Wang A, Pedersen WR, et al. 4-year results of a randomized controlled trial of percutaneous repair versus surgery for mitral regurgitation. J Am Coll Cardiol. 2013;62(4):317-28.

3. Toggweiler S, Zuber M, Surder D, Biaggi P, Gstrein C, Moccetti T, Pasotti E, Gaemperli O, Faletra F, Petrova-Slater I, et al. Two-year outcomes after percutaneous mitral valve repair with the MitraClip system: durability of the procedure and predictors of outcome. Open Heart. 2014; (1):e000056.

4. Buzzatti N, De Bonis M, Denti P, Barili F, Schiavi D, Di Giannuario G, La Canna G, Alfieri $\mathrm{O}$. What is a "good" result after transcatheter mitral repair? Impact of 2+ residual mitral regurgitation. J Thorac Cardiovasc Surg. 2016;151(1):88-96.

5. Kim J, Alakbarli J, Palumbo MC, Xie LX, Rong LQ, Tehrani NH, Brouwer $L R$, Devereux RB, Wong SC, Bergman GW, et al. Left ventricular geometry predicts optimal response to percutaneous mitral repair via MitraClip: integrated assessment by two- and three-dimensional echocardiography. Catheter Cardiovasc Interv. 2019;93(6):1152-60.

6. Obadia JF, Messika-Zeitoun D, Leurent G, lung B, Bonnet G, Piriou N, Lefevre T, Piot C, Rouleau F, Carrie D, et al. Percutaneous repair or medical treatment for secondary mitral regurgitation. N Engl J Med. 2018;379(24):2297-306.

7. Stone GW, Lindenfeld J, Abraham WT, Kar S, Lim S, Mishell JM, Whisenant B, Grayburn PA, Rinaldi M, Kapadia SR, et al. Transcatheter mitral-valve repair in patients with heart failure. N Engl J Med. 2018;379:2307.
8. Zhang Y, Wang WY, Morgan AE, Kim J, Handschumacher MD, Moskowitz CS, Levine RA, Liang G, Guccione JM, Weinsaft JW, et al. Mechanical effects of Mitraclip on leaflet stress and myocardial strain in functional mitral regurgitation - A finite element modeling study. PLOS:One. 2019;14:e0223472.

9. Palmieri V, Dahlof B, DeQuattro V, Sharpe N, Bella JN, de Simone G, Paranicas M, Fishman D, Devereux RB. Reliability of echocardiographic assessment of left ventricular structure and function: the PRESERVE study. Prospective randomized study evaluating regression of ventricular enlargement. J Am Coll Cardiol. 1999;34(5):1625-32.

10. Kim J, Di Franco A, Seoane T, Srinivasan A, Kampaktsis PN, Geevarghese A, Goldburg SR, Khan SA, Szulc M, Ratcliffe MB, et al. Right ventricular dysfunction impairs effort tolerance independent of left ventricular function among patients undergoing exercise stress myocardial perfusion imaging. Circ Cardiovasc Imaging. 2016;9(11):e005115.

11. Devereux RB, Alonso DR, Lutas EM, Gottlieb GJ, Campo E, Sachs I, Reichek N. Echocardiographic assessment of left ventricular hypertrophy: comparison to necropsy findings. Am J Cardiol. 1986;57(6):450-8.

12. Kim J, Rodriguez-Diego S, Srinivasan A, Brown RM, Pollie MP, Di Franco A, Goldburg SR, Siden JY, Ratcliffe MB, Levine RA, et al. Echocardiography-quantified myocardial strain-a marker of global and regional infarct size that stratifies likelihood of left ventricular thrombus. Echocardiography. 2017;34(11):1623-32.

13. Volo SC, Kim J, Gurevich S, Petashnick M, Kampaktsis P, Feher A, Szulc M, Wong FJ, Devereux RB, Okin PM, et al. Effect of myocardial perfusion pattern on frequency and severity of mitral regurgitation in patients with known or suspected coronary artery disease. Am J Cardiol. 2014;114(3):355-61.

14. Jones EC, Devereux RB, Roman MJ, Liu JE, Fishman D, Lee ET, Welty TK, Fabsitz RR, Howard BV. Prevalence and correlates of mitral regurgitation in a population-based sample (the strong heart study). Am J Cardiol. 2001;87(3):298-304.

15. Zoghbi WA, Adams D, Bonow RO, Enriquez-Sarano M, Foster E, Grayburn PA, Hahn RT, Han Y, Hung J, Lang RM, et al. Recommendations for noninvasive evaluation of native valvular regurgitation: a report from the American Society of Echocardiography Developed in Collaboration with the Society for Cardiovascular Magnetic Resonance. J Am Soc Echocardiogr. 2017;30(4):303-71.

16. Gelsomino S, van Garsse L, Luca F, Lorusso R, Cheriex E, Rao CM, Caciolli S, Vizzardi E, Crudeli E, Stefano P, et al. Impact of preoperative anterior leaflet tethering on the recurrence of ischemic mitral regurgitation and the lack of left ventricular reverse remodeling after restrictive annuloplasty. J Am Soc Echocardiogr. 2011;24(12):1365-75.

17. Sorajja P, Mack M, Vemulapalli S, Holmes DR Jr, Stebbins A, Kar S, Lim DS, Thourani V, McCarthy P, Kapadia S, et al. Initial experience with commercial transcatheter mitral valve repair in the United States. J Am Coll Cardiol. 2016;67(10):1129-40.

18. Oguz D, Eleid MF, Dhesi S, Pislaru SV, Mankad SV, Malouf JF, Nkomo VT, Oh $J K$, Holmes DR, Reeder GS, et al. Quantitative three-dimensional echocardiographic correlates of optimal mitral regurgitation reduction during transcatheter mitral valve repair. J Am Soc Echocardiogr. 2019;508947317(19):30800-4.

19. Maisano F, Franzen O, Baldus S, Schafer U, Hausleiter J, Butter C, Ussia GP, Sievert H, Richardt G, Widder JD, et al. Percutaneous mitral valve interventions in the real world: early and 1-year results from the ACCESS-EU, a prospective, multicenter, nonrandomized post-approval study of the MitraClip therapy in Europe. J Am Coll Cardiol. 2013;62(12):1052-61.

20. Lubos E, Schluter M, Vettorazzi E, Goldmann B, Lubs D, Schirmer J, Treede H, Reichenspurner $\mathrm{H}$, Blankenberg $\mathrm{S}$, Baldus $\mathrm{S}$, et al. MitraClip therapy in surgical high-risk patients: identification of echocardiographic variables affecting acute procedural outcome. JACC Cardiovasc Interv. 2014;7(4):394-402.

21. Reichenspurner H, Schillinger W, Baldus S, Hausleiter J, Butter C, Schaefer U, Pedrazzini G, Maisano F. Clinical outcomes through 12 months in patients with degenerative mitral regurgitation treated with the MitraClip(R) device in the ACCESS-EUrope phase I trial. Eur J Cardiothorac Surg. 2013;44(4):e280-8.

22. Nickenig G, Estevez-Loureiro R, Franzen O, Tamburino C, Vanderheyden M, Luscher TF, Moat N, Price S, Dall'Ara G, Winter R, et al. Percutaneous mitral valve edge-to-edge repair: in-hospital results and 1-year follow-up of 628 patients of the 2011-2012 pilot European sentinel registry. J Am Coll Cardiol. 2014;64(9):875-84.

\section{Publisher's Note}

Springer Nature remains neutral with regard to jurisdictional claims in published maps and institutional affiliations. 